\title{
3D-PHARMACOPHORE MODELLING OF OMEGA-3 DERIVATIVES WITH PEROXISOME PROLIFERATOR-ACTIVATED RECEPTOR GAMMA AS AN ANTI-OBESITY AGENT
}

\author{
IDA MUSFIROH ${ }^{1 *}$, GINNA MEGAWATI ${ }^{2}$, DEWI MARHENI DIAH HERAWATI ${ }^{2}$, AGUS RUSDIN ${ }^{3}$
}

${ }_{1}^{1}$ Department of Pharmaceutical Analysis and Medicinal Chemistry, Faculty of Pharmacy, Universitas Padjadjaran, Jatinangor 45363, Indonesia, ${ }^{2}$ Department of Public Health, Division of Medical Nutrition, Faculty of Medicine, Universitas Padjadjaran, Bandung 40161, Indonesia, ${ }^{3}$ Department of Pharmacy, Poltekkes Kemenkes Bandung, Bandung 40161, Indonesia *Email: ida.musfiroh@unpad.ac.id

Received: 22 Aug 2021, Revised and Accepted: 05 Oct 2021

\section{ABSTRACT}

Objective: The aim of this work was to study the pharmacophore model of omega-3 derivatives with the PPAR- $\gamma$ receptor using LigandScout 4.4 .3 to investigate the important chemical interactions of complex structure.

Methods: The methods consisted of structure preparation of nine chemical compounds derived from omega-3 fatty acids, database preparation creating 3D Pharmacophore modelling, validation pharmacophore, and screening test compounds.

Results: The result of the research showed that the omega-3 derivatives docosahexaenoic acid (DHA), when eicosapentaenoic acid (HPA), and docosapentaenoic acid (DPA) have the best pharmacophore fit values of 36.59; 36.56; and 36.56, respectively. According to the results of the pharmacophore study, the carbonyl and hydroxyl of the carboxylate functional groups become the active functional groups that exhibit hydrogen bonding interactions. While the alkyl chain (Ethyl and methyl groups) was the portion that can be modified to increase its activity.

Conclusion: Omega-3 derivatives could be used as a lead drug for the powerful PPAR- $\gamma$ receptor in the prevention and treatment of obesity.

Keywords: 3d-Pharmacophore modelling, Omega-3 derivatives, PPAR- $\gamma$, and Obesity

(C) 2021 The Authors. Published by Innovare Academic Sciences Pvt Ltd. Thisis an open access article under theCCBYlicense (https://creativecommons.org/licenses/by/4.0/) DOI: https://dx.doi.org/10.22159/ijap.2021.v13s4.43851 Journal homepage: https://innovareacademics.in/journals/index.php/ijap

\section{INTRODUCTION}

Obesity is one of the most common diseases, defined by a considerable expansion and alteration of adipose tissue in the body. Obesity has also been linked to the pathogenesis of the metabolic syndromerelated cardiovascular disease, which is the leading cause of mortality worldwide [1, 2]. Nowadays, the treatment and prevention of obesity disease have been done by behavioral therapy, pharmacological treatment, and surgical intervention. Although, there are many side effects which can reduce the quality of life. In light of this, the usage of natural substances is the most viable alternative [3].

Omega-3 is an essential nutrient that has been shown to assist lose weight by lowering the accumulation of body fat. Omega- 3 fatty acids play an important role in controlling lipid metabolism and acting as anti-inflammatory sensors. Although the method for preventing obesity comorbidity is unknown, it has been found to reduce insulin resistance, which is linked to obesity-related metabolic diseases, by binding to the protein peroxisome proliferator-activated receptor PPAR- $\gamma$ [4].

The Peroxisome Proliferator-Activated Receptors (PPARs) family regulates adipocyte differentiation, lipids, insulin sensitivity, and glucose homeostasis. PPAR- $\gamma$, which actively acts on adipose tissue and macrophages, triggers the differentiation of fat cells and regulates fatty acid storage and glucose metabolism by influencing related genes. Some anti-obesity medications that target PPAR- $\gamma$ have full agonist activity, which is associated with a high risk of cardiovascular adverse effects [5, 6].

We had conducted a molecular docking study of Omega-3 derivatives compounds with Peroxisome Proliferator-Activated Receptor Gamma (PPAR- $\gamma$ ) in a prior project. Based on the lowest binding energy, type of amino acid residue, and inhibition constant, we discovered that Docosahexaenoic acid has the best activity [7]. Also, because docosahexaenoic acid has only partial agonist action, it is assumed that it has no adverse effects on the cardiovascular system [7]. Although due to the lack of a detailed explanation of the molecular interaction between the drug and the receptor, this discovery remains uncertain. In addition, the functional groups that interact with the receptor are not determined in detail.
Therefore, the aim of this work was to performed the ligand-based drug design study of the pharmacophore model of omega-3 derivatives with the Proliferator-Activated Receptor Gamma (PPAR- $\gamma$ ) using LigandScout 4.4.3 to investigate the important chemical interactions of complex structure.

\section{MATERIALS AND METHODS}

\section{Structure preparation}

Nine chemical compounds derived from omega-3 fatty acids were chosen based on previous research concerning their bioactivity and pharmacological characteristics. The omega- 3 derivatives that were chosen are as follows: hexadecatrienoic acid (HTA), alpha-linolenic acid (ALA), stearidonic acid (SDA), eicosatrienoic acid (ETE), eicosatetraenoic acid (ETA), eicosapentaenoic acid (EPA), heneicosapentaenoic acid (HPA), docosapentaenoic acid (DPA), and docosahexaenoic (DHA). The 2D structures were generated with the ChemDraw 2D Ultra 12.0 program, and the energy was minimized using MM2 by ChemDraw 3D software, then all of the structure was saved with (.pdb) format [8]. The 2D Molecular Structure of Omega-3 Derivatives can be seen in fig. 1

\section{Database preparation}

Several databases are required for pharmacophore modeling, including the Active compound database, Decoy database, and test compound database. The test compounds were obtained through the previous preparation process, whereas the active and decoy compounds were downloaded from http://dude.docking.org/. Then, using LigandScout 4.4 .3 , each one was opened with the type of "training" compound for Active and Decoy, and the type of "test" for the test compound. The databases are then saved in. ldb format [9].

\section{Creating pharmacophore}

Ligand Scout 4.4.3 was used to perform pharmacophore modeling. The active compound database file that was previously prepared was opened and then sorted by cluster on the ligand-based menu. Each cluster is made up of one or more compounds, one of which must be converted into a training compound for each cluster, while the others are changed to the type "ignored." The pharmacophore model was then created, and the top ten pharmacophore models were validated [9]. 


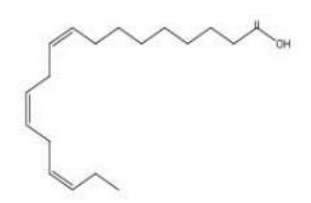

Alpha-linolenic acid

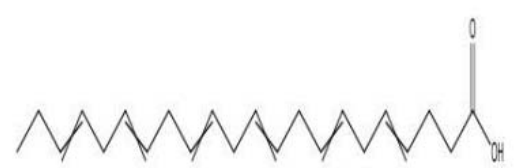

Docosahexaenoic acid
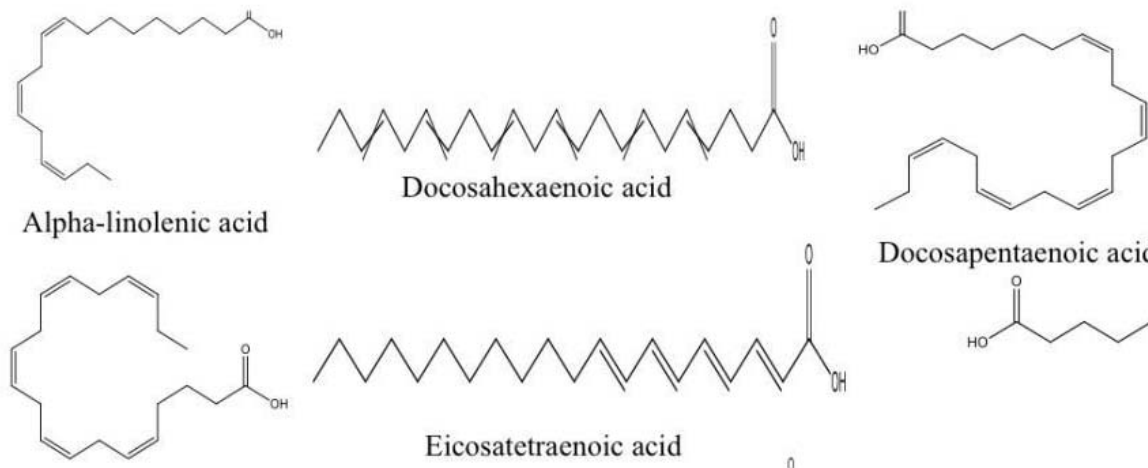

Docosapentaenoic acid

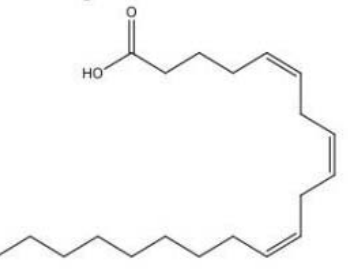

Eicosapentaenoic acid

Eicosatetraenoic acid

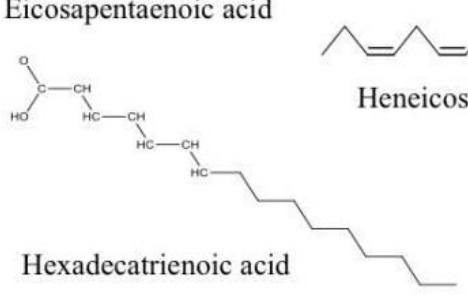

Eicosatrienoic acid

Fig. 1: The 2D molecular structure of omega-3 derivates

\section{Validation pharmacophore}

The ten pharmacophore models that were obtained were tested one by one to determine which one was the best. In the screening column, each pharmacophore model is entered, as well as the database for active and decoy compounds. Then click the "screening perspective" to perform screening pharmacophores. The Receiver Operating Characteristic (ROC) curve was used to assess the validity of the pharmacophores [9].

\section{Screening test compounds}

The test compound database that was previously generated is loaded in the screening column. In the ligand-based section, the best pharmacophore model was then sent to the screening column for further processing to determine the best compound based on the highest pharmacophore fit score [9].

\section{RESULTS}

The ROC curve was used to assess the validity of the pharmacophore. The trade-off between sensitivity (or TPR) and specificity (1-FPR) is depicted by the ROC curve. Classifiers with curves that are closer to the top-left corner perform better. The test becomes less accurate when the curve approaches the ROC space's 45-degree diagonal [10]. From the study, we had founded that pharmacophore model 4 has best ROC curve value.

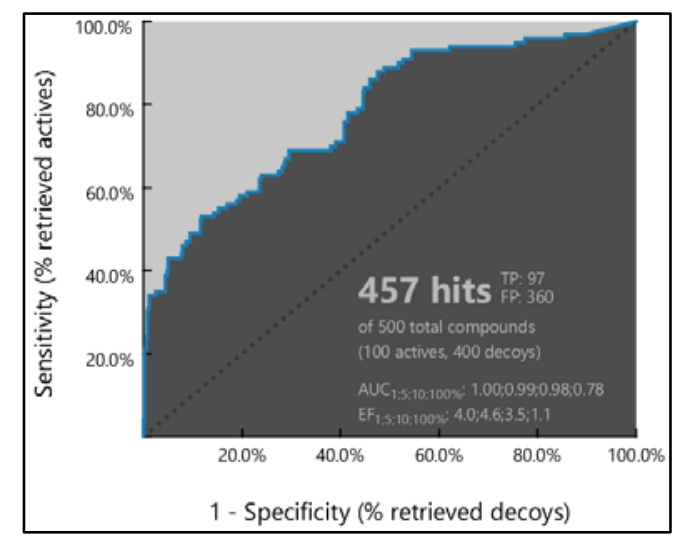

Fig. 2: The 3D structure-based pharmacophore model was validated using a Receiver Operating Characteristic (ROC) curve, with a set of 100 PPAR- $\gamma$ active and 400 decoy compounds

Table 1: LigandScout pharmacophore fit score of Omega-3 derivatives retrieved using the 3D-structure-based pharmacophore derived from propionic acid bound to the peroxisome Proliferator-activated receptor gamma

\begin{tabular}{lll}
\hline Compound & Pharmacophore-fit score & Binding energy (kcal/mol) \\
\hline Docosahexaenoic & 36.59 & -11.31 \\
Docosapentaenoic & 36.56 & $-11,01$ \\
Eicosapentaenoic & 36.56 & -10.82 \\
Hexadecatrienoic & 35.45 & -9.56 \\
Heneicosapentaenoic & 35.28 & -10.98 \\
Stearidonic & 35.27 & -10.09 \\
Alpha Linolenic & 35.08 & -10.07 \\
Eicosatetraenoic & 35.02 & -10.50 \\
Eicosatrienoic & 34.46 & -10.70 \\
\hline
\end{tabular}

The nine compounds of Omega-3 derivatives were screened for pharmacophore similarity to the best pharmacophore model (model 4), which is a pharmacophore composed of compounds that have been shown to have activity targeting PPAR- $\gamma$ receptors. The activity was evaluated based on the pharmacophore fit value. A higher fit score indicates a better fit to the model. From this study, docosahexaenoic (DHA), docosapentaenoic (DPA), and eicosapentaenoic (EPA) have the highest pharmacophore fit value. 


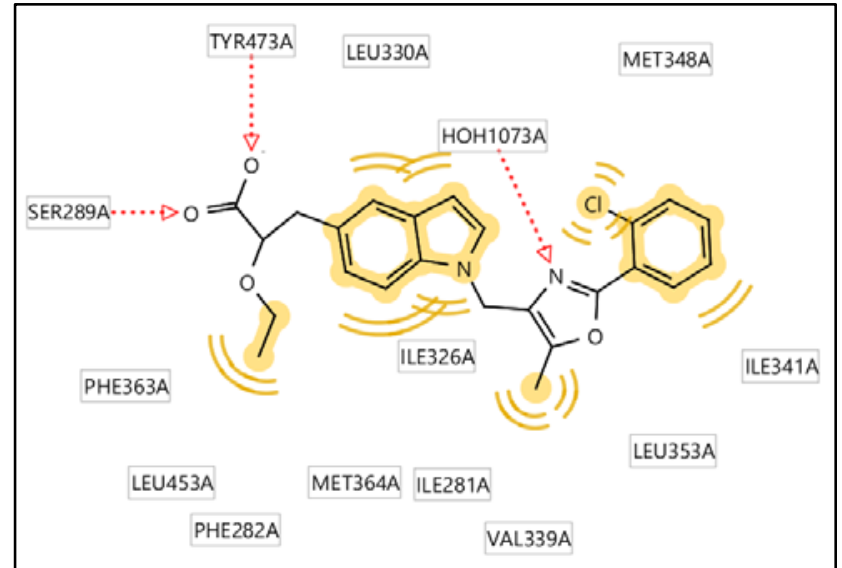

$3 \mathrm{~A}$

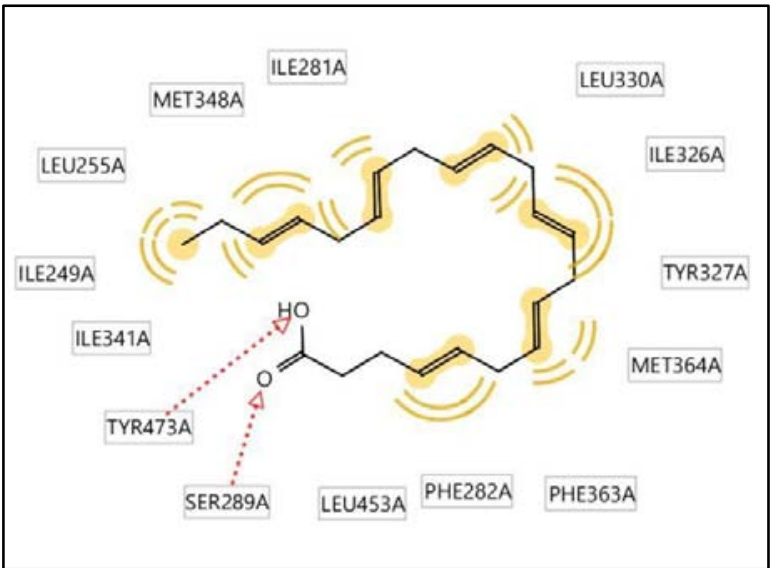

3B

Fig. 3: 3A, 2D-Pharmacophore model of propionic acid (Parent Compound) as a lead compound with the peroxisome proliferatoractivated receptor gamma (PPAR- $\gamma$ ); 3B: 2D-pharmacophore model of docosahexaenoic acid with the peroxisome proliferator-activated receptor gamma (PPAR- $\gamma)$

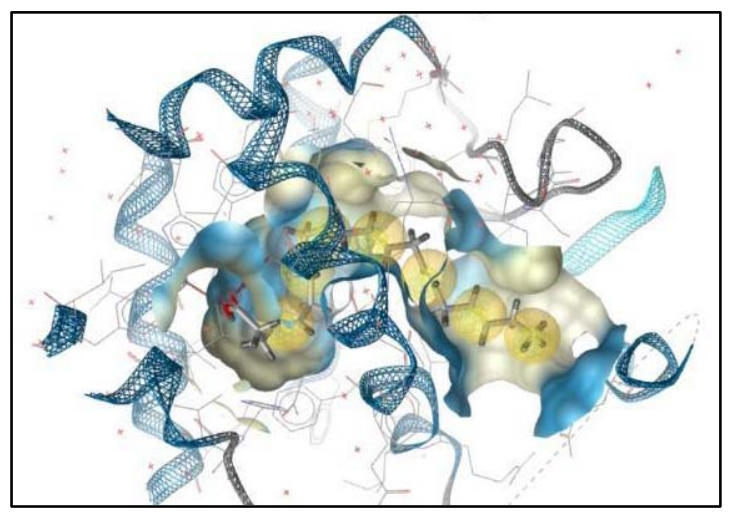

Fig. 4: 3D-Pharmacophore model of docosahexaenoic acid with the peroxisome proliferator-activated receptor gamma (PPAR- $\gamma$ )

In a molecular interaction study, the arrangement of functional groups that act as active sites of a structure was studied and assessed against their role in interacting with receptors. The angle and distance to the confirmation of the functional groups that make up a molecule have a significant effect on the ability to interact with receptors [11-13]. By comparing the results of pattern interaction between docosahexaenoic acid and propionic acid, we found that both have a similar amino acid residue on the carboxylic acid functional group, which can be seen on fig. $3 \mathrm{~A}$ and $3 \mathrm{~B}$; these compounds interact with TYR473A and SER289A amino acid residue.

\section{DISCUSSION}

At the beginning with pharmacophore validation, the ROC curve values were derived based on the validation findings of the 10 best pharmacophore models $(0.65,0.61,0.78,0.66,0.54,0.75,0.75,0.66$, and 0.62 ), where these results demonstrated that 3 out of 10 pharmacophores met the requirements. Models 4, 7, and 8 were validated ( $\geq 0.7)$. Model 4 was chosen because it had the greatest ROC curve value (AUC: 0.78 ), indicating that the pharmacophore model was able to properly distinguish actual active from decoy PPAR- $\gamma$ molecules in accordance with Kirchmair's methods [14]. The ROC curve value can be seen in fig. 2 .

In addition, as a result of pharmacophore fit value, compounds that fit the pharmacophore model should likewise have PPAR- $\gamma$ activity. Because not all of the model's features could be matched, throughout the virtual screening process, two features could be excluded. In this instance, pharmacophore fit scores would be lower if features could not be matched. Interestingly, all of the derivatives had higher pharmacophore fit scores (34.46 to 36.59) than the parent compound, with docosahexaenoic (36.59) and docosapentaenoic (36.56) having the best pharmacophore fit scores with the lowest of binding energy $(-11.31$ and-11.01 kcal/mol, respectively), indicating that their chemical features aligned best with the features of the pharmacophore model [15]. A higher geometric alignment of the compound's characteristics to the 3Dpharmacophore model is indicated by a higher fit score. Each compound's binding energy is presented for comparison (table 1). The suitability of the pharmacophore features on the ligands makes it easy for these to interact, which is correlated with lower bonding energy values. Binding energy value describes how spontaneous an interaction will occur. The lower the binding energy, the lower the activation energy, implying that it does not need a lot of energy to create the contact system between the ligand and the receptor, resulting in a spontaneous reaction. In addition, the best four compounds based on the fit-pharmacophore value have a correlation with the binding energy value, with the highest fitpharmacophore value having the best binding energy.

Furthermore, pharmacophore Modelling and Molecular Interaction Propionic acid (the parent compound) was employed as a lead compound or a comparative for Omega-3 derivatives in this investigation. Propionic acid was chosen as the lead compound since it has been shown to interact with Peroxisome ProliferatorActivated Receptors in prior investigations. Furthermore, the Nmethylene-substituted indole 5-propionic acid provides a suitable bio-isosteric replacement for the known tyrosine-based scaffold in PPAR- $\gamma$. The carboxylate and nitrogen groups in oxazole from Propionic Acid become acceptors of hydrogen bonding interactions, which interact with amino acids SER289A, TYR473A, and HOH1073A, as shown by pharmacophore modeling studies (fig. 3-4) [16].

This result is analogous to the hydrogen bonding interactions formed in Omega-3 derivative compounds (docosahexaenoic acid), where the carboxylic groups' hydroxy $(\mathrm{OH})$ and carbonyl $(\mathrm{C}=\mathrm{O})$ interactions function as donors and acceptors of hydrogen bonding interaction. And because of amino acids bound in TYR473A and SER289A are similar with the hydrogen bonding interactions that are presence in propionic acid-PPAR- $\gamma$ interaction and also same of functional group (Hydroxy carbonyl from carboxylic acid), this indicates that Docosahexaenoic Acid has the same mechanism of action [17]. Based on these results that the lead compound (Propionic Acid) has one sort of hydrogen bonding contact, namely the nitrogen atom in oxazole with the amino acid HCH1073A, which Docosahexaenoic Acid does not, but by considering two types of amino acid residues in the hydrogen bonding interactions. As well as, docosahexaenoic acid can be considered to have the same action 
as other identical amino acids due to have a comparable with hydrophobic interaction (LEU330A, MET38A, ILE341A, ILE281A, ILE362A, MET364A, PHE282A, LEU453A, and PHE363A). The fact that these two drugs have a comparable dominant interaction implies that they are positioned at the same active site. The carboxylate group is a pharmacophore structure that plays a key role in interacting with the PPAR- $\gamma$ receptor, as seen in the two structures.

\section{CONCLUSION}

The carbonyl and hydroxyl of the carboxylate functional groups from docosahexaenoic acid become the active functional groups that exhibit hydrogen bonding interactions, according to the results of the pharmacophore study. The alkyl chain (the ethyl and methyl groups) in docosahexaenoic Acid is the part that can be modified to boost activity. The fact that docosahexaenoic acid type of interaction is identical that occurs in the carboxylate group, both on the parent compound and on docosahexaenoic Acid with the same amino acid residues on TYR473A and SER289A. It suggests that docosahexaenoic acid could be used as a potential drug for the powerful PPAR- $\gamma$ receptor in the prevention and treatment of obesity.

\section{ACKNOWLEDGMENT}

This research was supported by Universitas Padjadjaran of Internal Grant through RDPD 2021

\section{FUNDING}

Nil

\section{AUTHORS CONTRIBUTIONS}

All the authors have contributed equally.

\section{CONFLICTS OF INTERESTS}

Declared none

\section{REFERENCES}

1. Ginsberg HN, MacCallum PR. The obesity, metabolic syndrome, and type 2 diabetes mellitus pandemic: Part I. Increased cardiovascular disease risk and the importance of atherogenic dyslipidemia in persons with the metabolic syndrome and type 2 diabetes mellitus. J Cardiometab Syndr. 2009;4(2):113-9. doi: 10.1111/j.1559-4572.2008.00044.x, PMID 19614799.

2. Koliaki C, Liatis S, Kokkinos A. Obesity and cardiovascular disease: revisiting an old relationship. Metabolism. 2019;92:98107. doi: 10.1016/j.metabol.2018.10.011, PMID 30399375.

3. Morgan PJ, Young MD, Lloyd AB, Wang ML, Eather N, Miller A, Murtagh EM, Barnes AT, Pagoto SL. Involvement of fathers in pediatric obesity treatment and prevention trials: A systematic review. Pediatrics. 2017;139(2):1-18. doi: 10.1542/peds.20162635, PMID 28130430

4. Calder PC. Very long-chain n-3 fatty acids and human health: fact, fiction and the future. Proc Nutr Soc. 2018;77(1):52-72. doi: 10.1017/S0029665117003950, PMID 29039280.
5. Senthil R, Sakthivel M, Usha S. Structure-based drug design of peroxisome proliferator-activated receptor-gamma inhibitors: ferulic acid and derivatives. J Biomol Struct Dyn. 2021;39(4):1295-311. doi: 10.1080/07391102.2020.1740790, PMID 32151198

6. Wang Y, Yao Y, Liu J, Wu L, Liu T, Cui J, Lee DY. Synthesis and biological activity of piperine derivatives as potential PPAR $\gamma$ agonists. Drug Des Devel Ther. 2020;14:2069-78. doi: 10.2147/DDDT.S238245, PMID 32546971.

7. Megawati G, Herawati DMD, Musfiroh I. Binding affinity of omega 3 fatty acid an agonist PPAR- $\gamma$ GPR120 receptor for obesity using molecular docking and ADME prediction. Eur J Mol Clin Med. 2021;7(10):1686-95

8. Deckelbaum LI, Isner JM, Donaldson RF, Laliberte SM, Clarke $\mathrm{RH}$, Salem DN. Use of pulsed energy delivery to minimize tissue injury resulting from carbon dioxide laser irradiation of cardiovascular tissues. J Am Coll Cardiol. 1986;7(4):898-908. doi: 10.1016/s0735-1097(86)80355-2, PMID 3082956.

9. Seidel T, Bryant SD, Ibis G, Poli G, Langer. 3D pharmacophore modeling techniques in computer-aided molecular design using LigandScout. Tutor Chemoinform. 2017;281:279-309.

10. Zou KH, O'Malley AJ, Mauri L. Receiver-operating characteristic analysis for evaluating diagnostic tests and predictive models Circulation. 2007;115(5):654-7. 10.1161/circulationaha.105.594929, PMID 17283280.

11. Caporuscio F, Tafi A. Pharmacophore modelling: a forty-yearold approach and its modern synergies. Curr Med Chem. 2011;18(17):2543-53. doi: 10.2174/092986711795933669, PMID 21568893

12. Choudhury C, Narahari Sastry G. Pharmacophore modelling and screening: concepts, recent developments and applications in rational drug design. Challenges and Advances in Computational Chemistry and Physics; 2019. p. 25-53. doi: 10.1007/978-3-030-05282-9_2.

13. Voet A, Zhang KY. Pharmacophore modelling as a virtual screening tool for the discovery of small-molecule proteinprotein interaction inhibitors. Curr Pharm Des. 2012;18(30):4586-98. doi: 10.2174/138161212802651616, PMID 22650262.

14. Kirchmair J, Distinto S, Markt P, Schuster D, Spitzer GM, Liedl KR, Wolber G. How to optimize shape-based virtual screening: choosing the right query and including chemical information. J Chem Inf Model. 2009;49(3):678-92. doi: 10.1021/ci8004226, PMID 19434901.

15. Seidel T, Ibis G, Bendix F, Wolber G. Strategies for 3D pharmacophore-based virtual screening. Drug Discov Today Technol. 2010;7(4):e221-8. doi: 10.1016/j.ddtec.2010.11.004

16. Kuhn B, Hilpert H, Benz J, Binggeli A, Grether U, Humm R, Marki HP, Meyer M, Mohr P. Structure-based design of indole propionic acids as novel PPARalpha/gamma co-agonists. Bioorg Med Chem Lett. 2006;16(15):4016-20. doi: 10.1016/j.bmcl.2006.05.007, PMID 16737814.

17. Chang S, Jiao X, Li CH, Gong XQ, Chen WZ, Wang CX. Amino acid network and its scoring application in protein-protein docking. Biophys Chem. 2008;134(3):111-8. doi: 10.1016/ j.bpc.2007.12.005, PMID 18329160. 